\title{
Genotypic Differences in Response to Hygromycin Effect on Untransformed Calli Death and Rice Germination
}

\author{
Shahanaz Sultana ${ }^{1}$, Chai-Ling Ho${ }^{1}$, Parameswari Namasivayam ${ }^{1}$ and Suhaimi Napis ${ }^{*}$
}

\begin{abstract}
Hygromycin is an efficient selective agent in transformation studies of wide ranges of crop. In this study, different concentrations of hygromycin were used to observe the effect on untransformed calli death, percent germination and seedling growth of three rice varieties (Oryza sativa L.) viz BRRI dhan29, MR219 and Taipei309. Hygromycin killed the untransformed calli and inhibited the germination of tested varieties in a concentration dependent manner. Among the tested varieties, the lowest and the highest calli death was observed in MR219 and Taipei309 respectively in all the concentrations of hygromycin. Whereas, the highest and the lowest percent germination were observed in MR219 and Taipei309 respectively. The minimal inhibitory concentration (MIC) for selection of calli were calculated as 42,40 and $47 \mathrm{mg} / \mathrm{L}$ hygromycin for BRRI dhan29, MR219 and Taipei309 respectively. During germination, 35, 62 and $32 \mathrm{mg} / \mathrm{L}$ hygromycin were suitable for the selection of BRRI dhan29, MR219 and Taipei 309 respectively. Shoot and root growth reduction after germination was increased with the increased concentration of hygromycin. Besides, root growth was more sensitive to hygromycin than the shoot. These results suggest that hygromycin increases calli death, decreases percent germination, and shoot and root growth in all varieties with the increasing rate of hygromycin. But these characteristics vary with different degrees in different genotypes as well as different stages.
\end{abstract}

Key words: hygromycin, calli, death, germination, rice

\section{INTRODUCTION}

Rice (Oryza sativa L.) is one of the major cereal crops throughout the world. However, the production and quality of rice are affected by different kinds of biotic and abiotic stresses. Therefore, the improvement of rice through genetic engineering or transformation provides an opportunity to introduce desired traits into rice. Agrobacterium-mediated transformation has been widely used in rice transformation (Hoque et al., 2005; Tyagi et al., 2007 and Sultana et al., 2012). The selectable marker gene that confers antibiotic resistant is generally incorporated together with the gene of interest to select the true transformants (Jenkins et al., 1995). In most of the rice transformation studies, hygromycin phosphotransferase was used as the selective agent for transformed plant (Gritz and Davies, 1983) because most monocot tissue showed a higher sensitivity to it (Yara et al., 2001). Hygromycin phosphotranferase (hpt) gene encodes a product, which allows the transformed cells to survive and grow under conditions that kill or restrict the growth of untransformed cells (Ortiz et al., 1996). This hpt inactivates the antibiotic hygromycin through phosphorylation. So, the selection of transformed cell is crucial for transformation studies because cells could be escaped from the effect of hygromycin due to rapid cell division. However, different concentrations of hygromycin, duration of selection period and genetic materials also influence the selection of transformed cells. For this reason, the experiment was conducted to observe the effect of hygromycin on untransformed calli, germination and seedling growth of three rice varieties (Oryza sativa L.) viz BRRI dhan29, MR219 and Taipei309. Taipei309 is a popular japonica variety used in tissue culture and transformation work. On the other hand, BRRI dnan29 (About BRRI (2014) and MR219 (MARDI, 2011) are high yielding rice varieties in Bangladesh and Malaysia respectively, which can be used as test materials to improve their tolerance to different stresses.

\footnotetext{
*Present address : Biotechnology Division, Bangladesh Rice Research Institute, Gazipur 1701, Bangladesh. ${ }^{1}$ Department of Cell and Molecular Biology, Faculty of Biotechnology and Bio-molecular Sciences, Universiti Putra Malaysia, Malaysia. ${ }^{1}$ Corresponding author's email: shahanaz.biotech@brri.gov.bd
} 
Callus and seed germination stages were selected for this study since these two stages are commonly used in transformation work for rice to screen the putative transformants. The information on suitable concentration of hygromycin for calli death and germination is essential, which can be used for selection of putative transformed calli and seedling of those particular rice varieties for introducing agronomically useful traits by using genetic engineering techniques. Therefore, the aim of this study was to investigate the effect of hygromycin on calli death and seed germination as well as to calculate the minimal inhibitory concentration during callusing and germination of three rice varieties.

\section{MATERIALS AND METHODS}

\section{Seed materials and culture media}

Matured seeds of rice (Oryza sativa L.) varieties BRRI dhan29 and Taipei309 were obtained from Bangladesh Rice Research Institute (BRRI), Bangladesh and MR219 was obtained from Malaysian Agricultural Research and Development Institute (MARDI), Malaysia. Murashige and Skoog (MS) (Murashige and Skoog, 1962) basal medium was used for callusing and seed germination.

\section{Calli induction and death}

Dehulled seeds of BRRI dhan29, MR219 and Taipei309 were surface sterilized and blotted dry with a sterilized filter paper. The sterilized seeds were inoculated on callus induction medium (MS basal medium with $2 \mathrm{mg} / \mathrm{L} \mathrm{2,4-D,}$ solidified with $0.4 \%$ gelrite) and cultured at $32^{\circ} \mathrm{C}$ for three weeks under light. The threeweek old matured calli were transferred onto MSO (basal MS medium without hormone) containing different concentrations of hygromycin $(0,20,30,40,50,60$ and $70 \mathrm{mg} / \mathrm{L}$ respectively). The calli were incubated for two weeks until they turned brown (cell death). Four replicates were carried out for each treatment with 30 calli in each replicate. Minimal inhibitory concentration (MIC) for hygromycin was determined at which $50 \%$ calli death could be induced.

\section{Germination of rice seeds and seedling growth reduction}

Another batch of sterilized seeds was plated onto MSO containing different concentrations of hygromycin B (0, 20, 30, 40, 50, 60 and $70 \mathrm{mg} / \mathrm{L}$ respectively). Seeds were incubated for 10 days and germination percentage, length of shoot and root were measured. Four replications were made for each treatment with 50 seeds for each of the replications. It was determined that MIC for hygromycin could reduce 50\% germination. Percent growth reduction (shoot and root) was calculated by using the following formula:

$$
\% \text { shoot/root growth reduction Shoot/root growth }(\mathrm{cm}) \text { under controlcondition - Shoot/root growth }(\mathrm{cm}) \text { under treated condition }
$$

\section{RESULTS}

\section{Effect of hygromycin}

On calli death: The effect of different concentrations of hygromycin on tested genotypes varied in a concentration dependent manner. After two weeks, $70 \mathrm{mg} / \mathrm{L}$ of hygromycin induced 89, 93.75 and 90\% calli death in BRRI dhan29, MR219 and Taipei309 respectively, whereas $60 \mathrm{mg} / \mathrm{L}$ of hygromycin induced $76.39,78.89$ and $70.5 \%$ calli death in BRRI dhan29, MR219 and Taipei 309 respectively. However, medium containing 20 $\mathrm{mg} / \mathrm{L}$ hygromycin did not show any effect on Taipei309 calli while 15.5 and $25 \%$ calli death were observed in BRRI dhan29 and MR219, respectively (Fig. 1). No calli death was observed in control treatment $(0 \mathrm{mg} / \mathrm{L}$ hygromycin). 


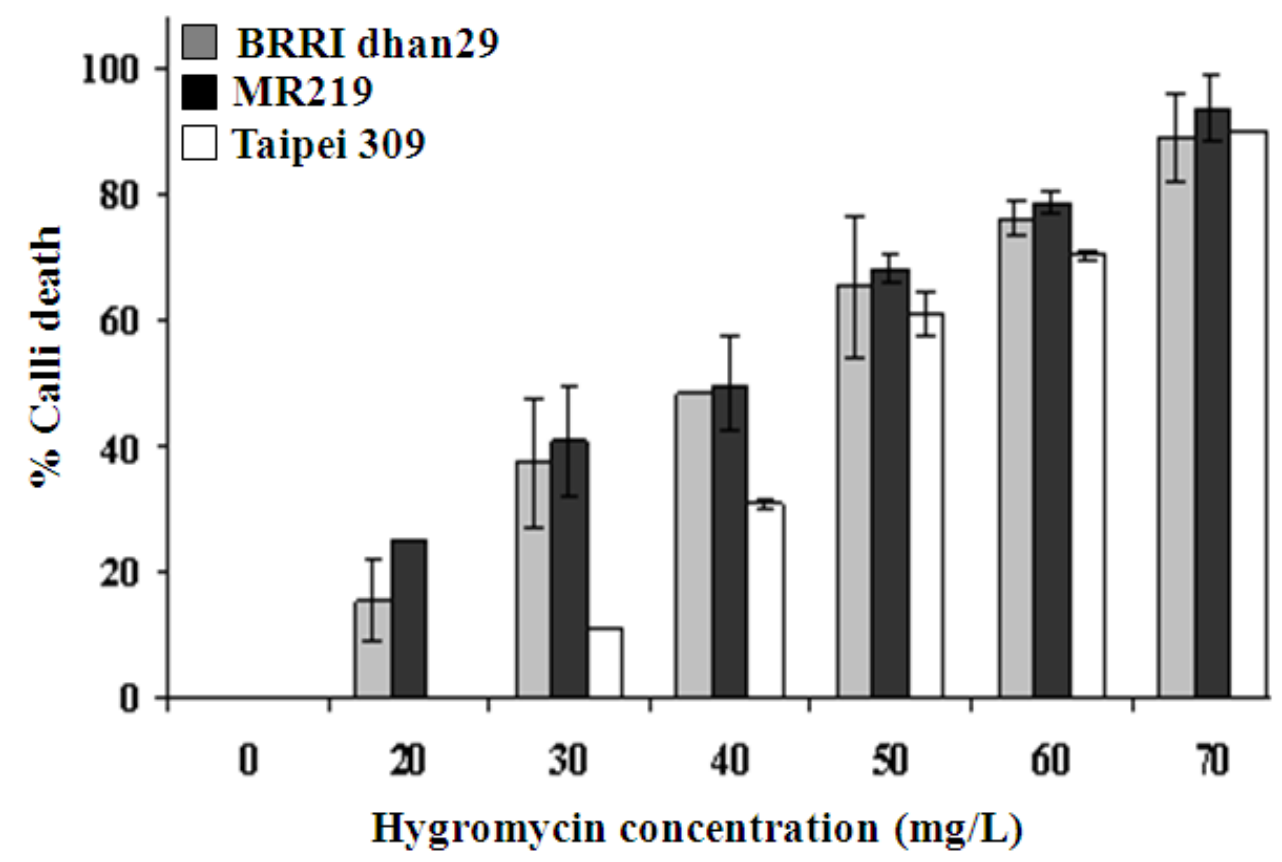

Fig. 1. Effect of hygromycin on calli death of different rice varieties

The y-axis shows means of three replication and vertical bars indicate SD.

On germination: Hygromycin inhibited the germination rate of seeds of all the tested genotypes. The germination rate declined with the increase of hygromycin concentration (Fig. 2). At $70 \mathrm{mg} / \mathrm{L}$ hygromycin $13.89,41.85$ and
$13.33 \%$ germination was observed in BRRI dhan29, MR219 and Taipei309 respectively. Whereas, more than $90 \%$ germination was observed in control treatment of all the tested varieties.

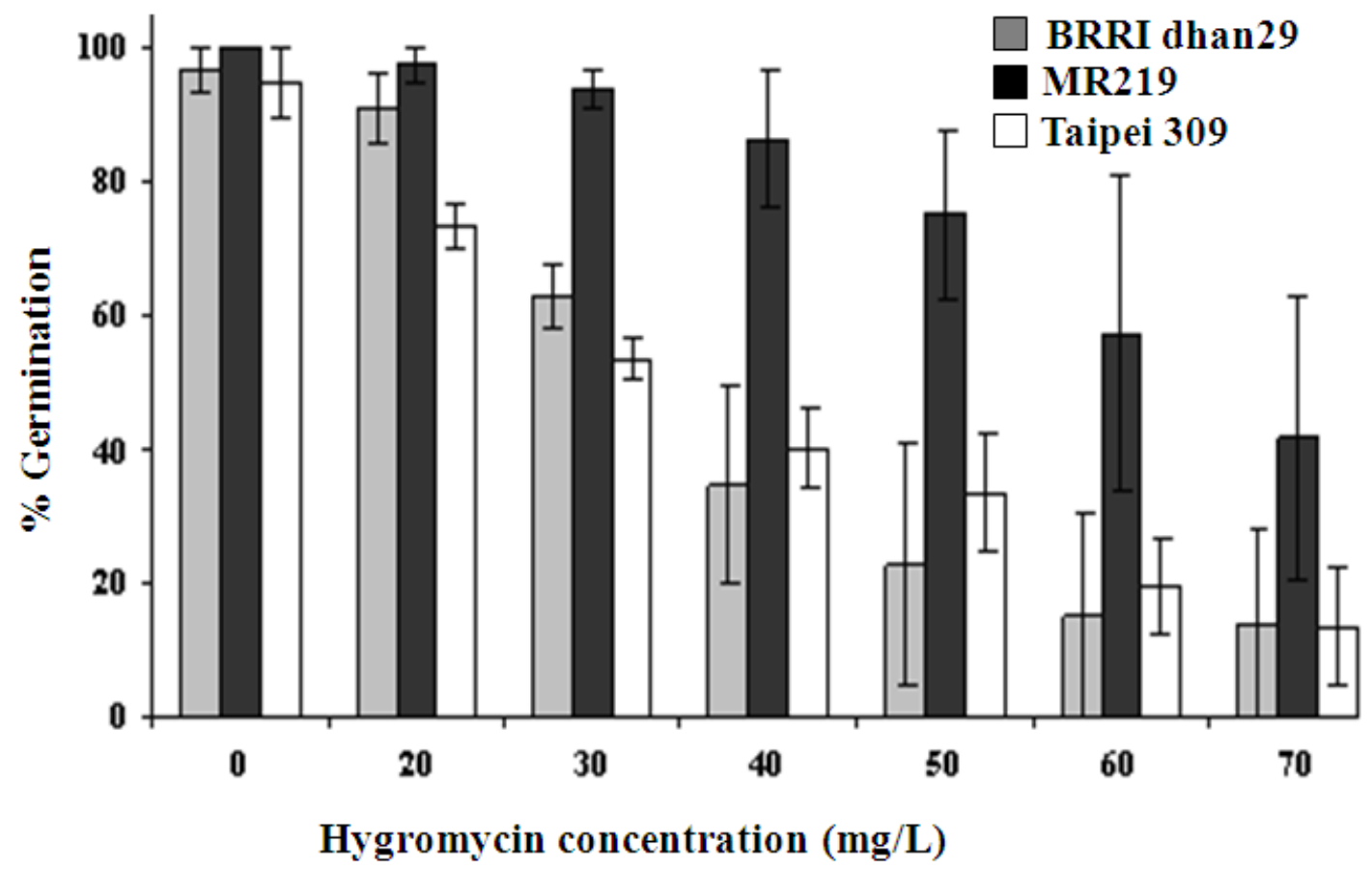

Fig. 2. Effect of different concentration of hygromycin on seed germination of three rice varieties The y-axis shows means of three replication and vertical bars indicate SD. 
On shoot and root growth. After 10 days on medium containing $20 \mathrm{mg} / \mathrm{L}$ of hygromycin, 43, 29.63 and $57.2 \%$ shoot reduction was observed in BRRI dhan29, MR219 and Taipei309 respectively compared to control treatment (Fig. 3a). At $30 \mathrm{mg} / \mathrm{L}$ hygromycin, more than $50 \%$ shoot growth reductions were observed in all the varieties. At $70 \mathrm{mg} / \mathrm{L}$ hygromycin concentration, very little shoot growth $(>90 \%$ reduction) was observed and most of the seedlings died after germination in all tested varieties (Fig. 3a). Root growth also declined with the increasing of concentration of hygromycin (Fig. 3b). Hygromycin suppressed the growth of primary root and lateral root. Lateral root growth was declined after $30 \mathrm{mg} / \mathrm{L}$ and higher concentration of hygromycin. At 70 $\mathrm{mg} / \mathrm{L}$ hygromycin concentration, no root growth was observed in all tested varieties (Fig. $4)$.
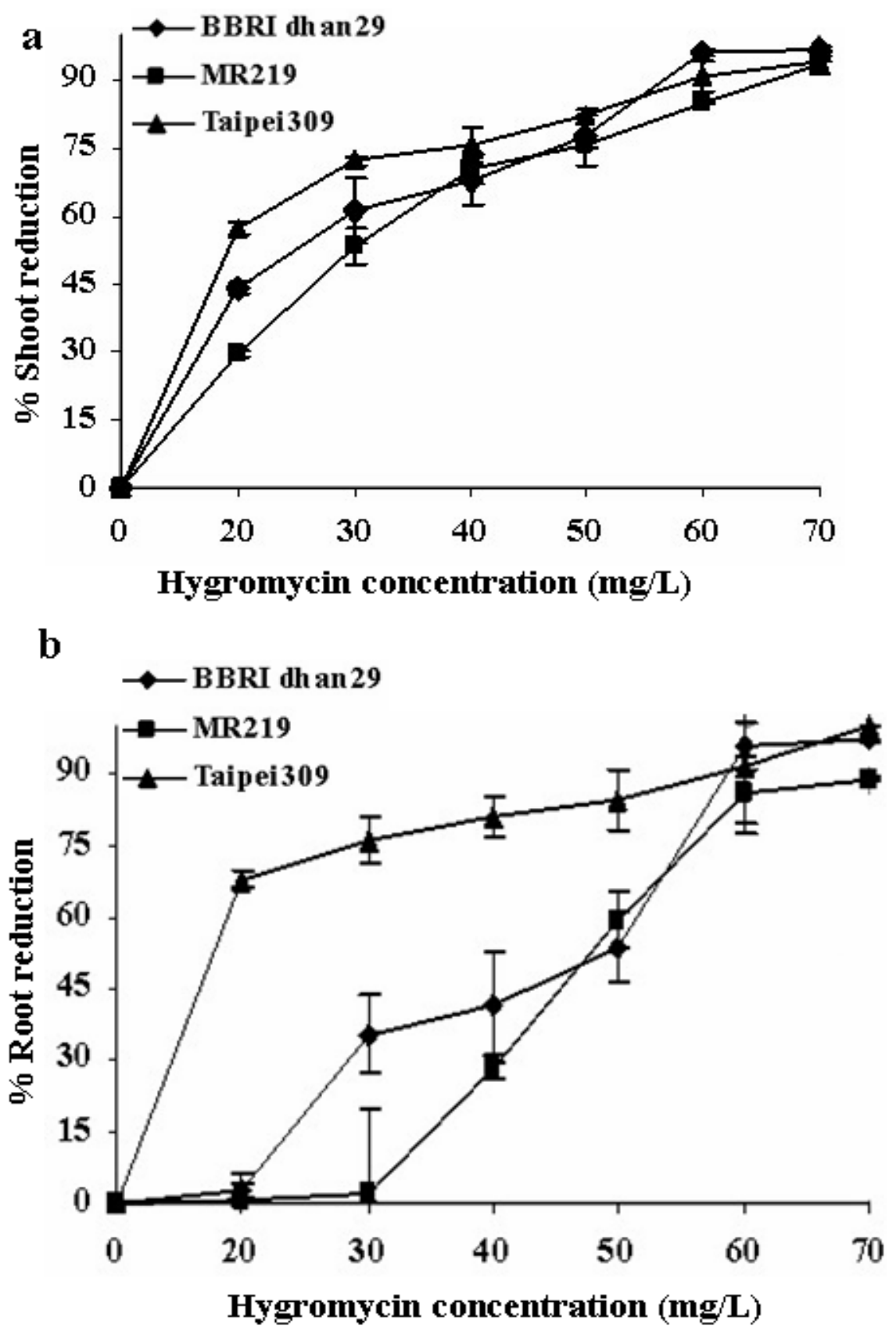

Fig. 3. Effect of different concentration of hygromycin on reduction of (a) shoot and (b) root length of three rice varieties. The $y$-axis shows mean $\%$ shoot and root reduction of three replication \pm SD 


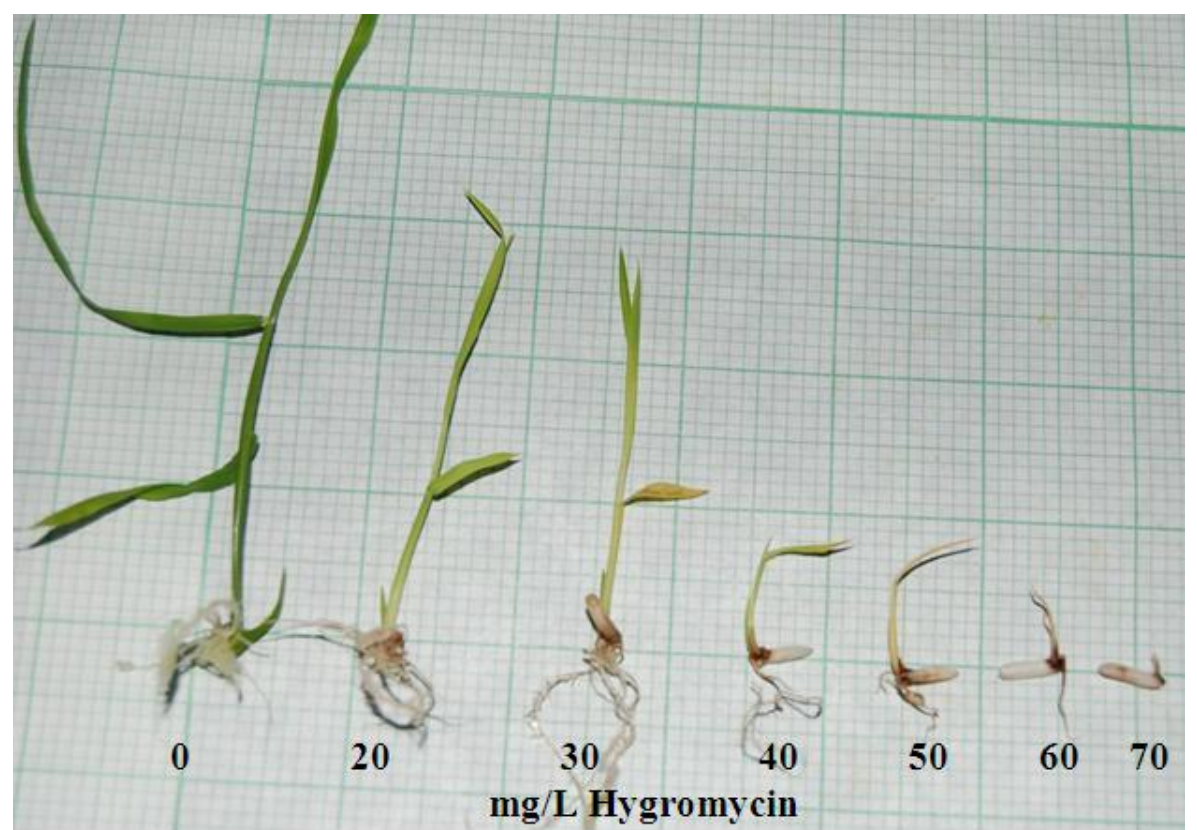

Fig. 4. Effect of hygromycin on shoot and root growth (BRRI dhan29). Seeds were germinated on MSO containing different concentration of Hygromycin.

MIC for selection of transgenic calli and plants Approximately $50 \%$ of calli death was observed at $42, \quad 40$ and $47 \mathrm{mg} / \mathrm{L}$ hygromycin concentrations in BRRI dhan29, MR219 and Taipei309 respectively. Based on the results, it is calculated that 42,40 and $47 \mathrm{mg} / \mathrm{L}$ hygromycin $B$ were suitable concentrations for primary selection of the putative transformed calli of BRRI dhan29, MR219 and Taipei309 respectively.

On the other hand, during germination $50 \%$ reduction was observed at 35,62 and $32 \mathrm{mg} / \mathrm{L}$ of hygromycin in comparison with the control treatment in BRRI dhan29, MR219 and Taipei309 respectively.

\section{DISCUSSIONS}

Transformation is a powerful tool to complement the conventional breeding methods in the genetic improvement of rice cultivar. Hygromycin plays an important role in the selection process of transformation studies since untransformed cells could be escaped from the effect of hygromycin due to rapid cell division. In this study, it was quite evident that the calli of three rice varieties responded differently to different hygromycin concentrations and the MIC for the selection of calli showed variation among the rice varieties. During callusing, calli of Taipei309 was not affected by $20 \mathrm{mg} / \mathrm{L}$ hygromycine while calli of BRRI dhan29 and MR219 showed $15.5 \%$ and $25 \%$ calli mortality. From the results obtained during callusing it was estimated that hygromycin concentration of 42,40 and $47 \mathrm{mg} / \mathrm{L}$ were suitable for primary selection of the calli of BRRI dhan29, MR219 and Taipei309 respectively. But the results were slightly dissimilar with the findings of Cheng et al. (1998) who reported that the presence of 50 $\mathrm{mg} / \mathrm{L}$ hygromycin throughout the callusing was good. This variation in sensitivity to hygromycin during callusing might be due to the use of different genotypes and explants, suggesting that different concentrations of hygromycin should be used for different explants and different genotypes. The response of specific genotypes to hygromycin is not only varied at different concentrations but also varied at different growth stages. Among the tested varieties, Taipei309 showed the highest tolerance to hygromycin during callusing but showed the lowest tolerance during germination, whereas MR219 showed higher tolerance during germination but the lowest during callusing. The variation of sensitivity to hygromycin during callusing and germination suggested that different selected concentrations of hygromycin should be applied at different stages of selection. Hygromycin not only reduced the germination but also influenced the shoot and root growth because it restricts the

Genotypic Differences in Response 42 
growth of untransformed cells (Ortiz et al., 1996). Similar findings were reported by Duan and Ding (2007) in which the growth and development of main root and lateral root of Arabidopsis seedling were affected by hygromycin might be due to restraining synthesis of some proteins. Thus, it is important to know the suitable concentration of hygromycin of respective genotype and stage of plants.

\section{CONCLUSIONS}

Hygromycin inhibited the growth of calli, restricted the germination and finally reduced the growth of shoot and root with the increasing rate of hygromycin concentration. But it varied with the genotypes and stage of plants.

\section{ACKNOWLEDGMENT}

Shahanaz Sultana was supported by the Third World Organization for Women in Science (TWOWS). Mature seeds of BRRI dhan29 and Taipei309 were obtained from the Bangladesh Rice Research Institute (BRRI), Bangladesh. MR219 obtained from Malaysian Agricultural Research and Development Institute (MARDI).

\section{REFERENCES}

About BRRI (Bangladesh Rice Research Institute). 2014. Bangladesh Rice Research Institute (BRRI), Publication no. 6,18

Cheng, X Y, R Sardana, H Kaplan and I Altosaar. 1998. Agrobacterium-transformed rice plants expressing synthetic cryIA(c) gene are highly toxic to striped stem borer and yellow stem borer. Proceedings of the National Academy of Sciences of the United States of America (PNAS) 95, 2767-2772.

Duan, H and X Ding. 2007. Effects of hygromycin on growth and development of arabidopsis seedling roots. Pakistan Journal of Botany 39: 6, 2167-2173.

Gritz, L and J Davies. 1983. Plasmid-encoded hygromycin B resistance: the sequence of hygromycin B phosphotransferase gene and its expression in Escherichia coli and Saccharomyces cerevisiae. Gene 25,179-188.

Hoque, M E, J W Mansfeld and H B Mark. 2005. Agrobacterium-mediated transformation of Indica rice genotypes: an assessment of factors a ecting the transformation e ciency. Plant cell, Tissue and Organ Culture 82, 45-55.

MARDI. 2011. http://agromedia.mardi.gov.my? magritech/tech_detail_fdcrop.phplid=346. Accessed on 13 December 2011.

Jenkins, J N, J C McCarty and T Wofford. 1995. Bt cotton: a new era in cotton production. Procedings Beltwide Cotton Conference, Vol 1, National Cotton Council. Memphis, TN, USA,171-173.

Murashige, $\mathrm{T}$ and F Skoog. 1962. A revised medium for rapid growth and bioaasays with tobacco tissue cultures. Plant Physiology 15, 473-497.

Ortiz, J P A, M I Reggiardo, R A Ravizzini, F Altabe, G D L Cervigni, M A Spitteler, M M Morata, F E Elias and R $\mathrm{H}$ Vallejos. 1996. Hygromycin resistance as an efficient selectable marker for wheat stable transformation. Plant Cell Reports 15, 877-881.

Sultana, S, C-Y Khew, M M Morshed, P Namasivayam, S Napis and C-L Ho. 2012. Overexpression of monodehydroascorbate reductase from a mangrove plant (AeMDHAR) confers salt tolerance on rice. Journal of Plant Physiology 169:3, 311-318.

Tyagi, H, S Rajasubramaniam and I Dasgupta. 2007. Regeneration and Agrobacterium-mediated transformation of a popular indica rice variety, ADT39. Current Science 93, 678-683.

Yara A, M Otani, K Kusumi, O Matsuda, T Shimada and K Iba. 2001. Production of transgenic japonica rice (Oryza sativa), cultivar Taichung65, by Agrobacterium-mediated transformation. Plant Biotechnology 18, 305-310. 